\title{
Bacterial Sepsis Pathogens and Resistance Patterns in a South Asian Tertiary Care Hospital
}

Zia U. Rehman ${ }^{1}$, Mohammad Hassan Shah ${ }^{2,}{ }^{3}$, Muhammad Nauman Shah Afridi ${ }^{4}$, Hafsa Sardar ${ }^{5}$, Ahmad Shiraz ${ }^{6}$

1. Nephrology and Kidney Transplant Unit, Rehman Medical Institute, Peshawar, PAK 2. Internal Medicine, Rehman Medical Institute, Peshawar, PAK 3. Haematology, King's College Hospital, London, GBR 4. General Surgery, Ayub Teaching Hospital, Abbottabad, PAK 5. Radiology, Hayatabad Medical Complex, Peshawar, PAK 6. General Surgery, Hayatabad Medical Complex, Peshawar, PAK

Corresponding author: Zia U. Rehman, zia.rehman026@gmail.com

\section{Abstract}

\section{Objective}

The aim of this study was to determine common microorganisms causing septicemia and their antimicrobial sensitivities in patients admitted to a tertiary care hospital.

\section{Methods}

A cross-sectional study was conducted using clinical criteria to diagnose patients as having septicemia where blood for culture and sensitivity (CS) was sent to the laboratory of a tertiary care hospital, Rehman Medical Institute, (Peshawar), Pakistan, during 2019. All patients diagnosed with septicemia regardless of age and gender were included in the study. The blood CS report was collected after 7-14 days of inoculation. Data were recorded on structured performa and analyzed using SPSS Version 20 (IBM Corp.).

\section{Results}

A total of 176 patients, with a mean age of $2.92 \pm 1.32$ years, fulfilled the criteria for sepsis with a mean age of $2.92 \pm 1.32$ years. Among them, $61.9 \%$ were male and $38.1 \%$ were female. Among common bacterial isolates, Staphylococcus aureus was found in $37.5 \%$ of samples followed by skin contaminants (18.2\%), methicillinresistant Staphylococcus aureus (MRSA) (14.8\%), and Escherichia coli in (11.4\% cases). None of the antibiotics had susceptibility of more than $60 \%$. Susceptibility to piperacillin/tazobactam and ampicillin/sulbactam was found in $21.5 \%$ and $14.6 \%$ of the samples, respectively, while in cephalosporins, cefoxitin's susceptibility was $28.5 \%$, whereas both ceftriaxone and cephazolin were equally effective in $19.4 \%$ cases. Furthermore, $38.9 \%$ of the samples were susceptible to ciprofloxacin and $24.3 \%$ to levofloxacin. The susceptibilities of amikacin and gentamicin in aminoglycosides were $56.3 \%$ and $47.2 \%$, respectively, while that of imipenem and meropenem were $59.7 \%$ and $22.9 \%$, respectively. Lastly, clindamycin had an efficacy in $42.4 \%$ of samples.

Review began 04/17/2021 Review ended 05/11/2021 Published 05/18/2021

\section{(c) Copyright 2021}

Rehman et al. This is an open access article distributed under the terms of the Creative Commons Attribution License CC-BY 4.0., which permits unrestricted use, distribution, and reproduction in any medium, provided the original author and source are credited.

\section{Conclusion}

The susceptibility of bacterial isolates in septicemia to common antibiotics was low, thus risking therapeutic failure in septic patients. Widespread resistance may be due to the excessive use along with over-thecounter availability of antibiotics, which therefore requires regulation as it is an alarming situation.

Categories: Internal Medicine, Pathology, Infectious Disease

Keywords: susceptibility, blood culture, resistant, sensitivity, histopathology \& microbiology, antibiotic

\section{Introduction}

Sepsis is a common problem associated with substantial mortality and significant consumption of healthcare resources [1]. It is the inflammatory response of the body toward the infectious agent, which, in turn, may cause severe sepsis and septic shock, which are its more severe forms [2]. Globally, the incidence of sepsis was 48.9 million in 2017, out of which 33.1 million had an underlying infectious disease and 15.8 million had other non-communicable diseases [3]. A greater part of the global sepsis burden is contributed by low- and middle-income countries, where $90 \%$ of sepsis deaths occur from pneumonia, meningitis, or other infections. The highest number of newborn and infant deaths occurring from sepsis is present in Asia and sub-Saharan Africa [4]. It has been reported that sepsis occurs in approximately $2 \%$ of all hospitalizations in developed countries; in intensive care units (ICUs), the prevalence ranged from $6 \%$ to $30 \%$ [5]. In Pakistan, the prevalence of septicemia due to bacteria was $14.75 \%$ [6]. An observational study in tertiary care hospitals reported that $40.7 \%$ of patients in tertiary care hospital ICUs had severe sepsis, while 59.3\% developed septic shock [7]. The most common isolated bacteria among patients admitted in ICUs of tertiary care units included Staphylococcus aureus (36.38\%) followed by Escherichia coli (18.28\%) and methicillin-resistant Staphylococcus aureus (MRSA; 7.0\%). Other bacteria included Streptococcus faecalis, 
Salmonella typhi, Pseudomonas species, and Candida species [6]. Overall mortality due to septic conditions is $12.5 \%$, but it varies (ranging from $5.6 \%$ to $34.2 \%$ ) by age and organ involvement [8]. Sepsis prevalence also varies by patient age. The majority of cases (58-65\%) occur in older age groups. [9]. Martin et al. reported a $26 \%$ higher risk of death in elderly patients with sepsis during the first week of hospitalization compared to younger patients [10]. Similarly, sepsis is the leading cause of death globally in infants and patients aged over 50 years [11]. Sepsis incidence among children is strikingly high in early childhood [3]. A hospital-based study in Pakistan showed that $25 \%$ of admitted neonates had sepsis [12].

Mortality in sepsis is increased with ineffective antibacterial therapy, which, in turn, is linked to bacterial multidrug resistance. Preceding empiric antibiotic prescriptions, incomplete courses of drugs, and poor nutritional status increase the risk [13]. Given these influences, regional variation in microbiology and resistance patterns is expected. The present study aims to define organisms responsible for bacterial sepsis, as well as their antibiotic resistance patterns, in different age groups of patients admitted to Rehman Medical Institute (RMI), Peshawar, Pakistan.

\section{Materials And Methods}

This cross-sectional study identified patients ranging from neonates to elderly with a maximum age of 82 years over a period of one year from May 2019 to May 2020 who met sepsis criteria by the 2017 National Institute for Health and Clinical Excellence Sepsis guidelines at RMI [14]. Blood culture results including susceptibilities, patient demographics, and medical history were recorded. Sensitivity patterns were analyzed by age group, organism, and underlying diseases. SPSS Version 20 (IBM Corp., Armonk, NY) was used for data analysis.

\section{Results}

A total of 176 patients fulfilled the clinical criteria for sepsis, of whom 109 (61.9\%) were male patients and 67 (38.1\%) were female patients, with a mean age of $2.92 \pm 1.32$ years. Among the isolated bacteria, Staphylococcus aureus was the most common (37.5\%) followed by skin contaminants (17.6\%) and MRSA (14.8\%), whereas Escherichia coli accounted for 11.4\% (Figure 1).

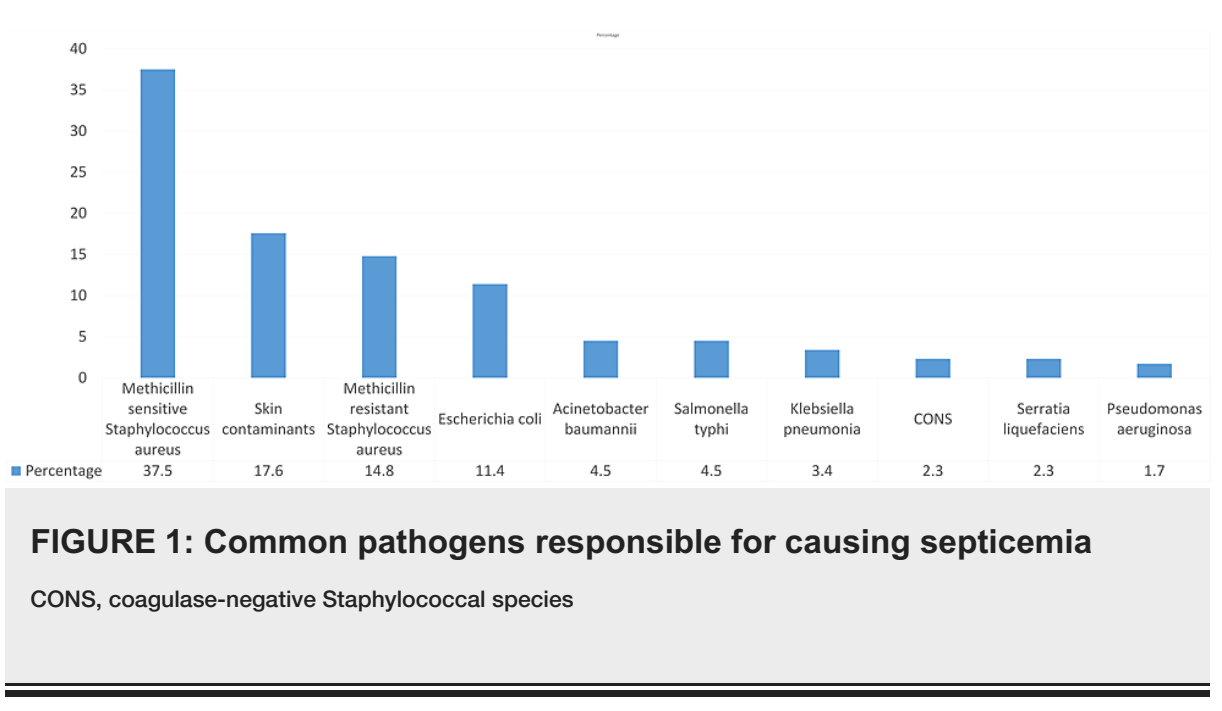

Of the 176 samples, 144 had clear results of sensitivity and resistance pattern, while 32 samples were deemed skin contaminants and excluded from further analysis, suggesting possible septicemia due to viral causes. In the penicillin group, only piperacillin/tazobactam and ampicillin/sulbactam had $21.5 \%$ and $14.6 \%$ susceptibilities, respectively. The susceptibility was very low for other penicillins. Among cephalosporins, cefoxitin was found effective in $28.5 \%$ of cases, while cephazolin and ceftriaxone both showed susceptibility in $19.4 \%$ of the cases. Among quinolones, $38.9 \%$ of the samples were susceptible to ciprofloxacin and $24.5 \%$ to levofloxacin, respectively. Among aminoglycoside, only amikacin and gentamicin were found effective in $56.3 \%$ and $47.2 \%$ of septicemia cases, respectively. Among carbapenems, imipenem and meropenem were found effective in $59.7 \%$ and $22.9 \%$ of the cases, respectively. Lastly, clindamycin showed susceptibility in $42.4 \%$ of samples.

In addition, susceptibility to vancomycin was $58.3 \%$ followed by chloramphenicol, which was found susceptible in $57.6 \%$ of the samples. Susceptibilities to other antibiotics included doxycycline (52.8\%), minocycline (47.2\%), co-trimoxazole (34.7\%), and rifampicin (31.2\%). On the other hand, very low susceptibilities were found for novobiocin, colistin, nalidixic acid, tigecycline, nitrofurantoin, and teicoplanin (Table 1). 


\section{Cureus}

\begin{tabular}{|c|c|c|c|}
\hline & $\mathbf{N}$ & Resistant, N (\%) & Susceptible, $\mathbf{N}(\%)$ \\
\hline \multicolumn{4}{|l|}{ Penicillin group } \\
\hline Ampicillin & 144 & $134(93.1 \%)$ & $10(6.9 \%)$ \\
\hline Amoxicillin/clavulanic acid & 144 & 135 (93.75\%) & $9(6.3 \%)$ \\
\hline Amoxicillin & 144 & 138 (95.8\%) & $6(4.2 \%)$ \\
\hline Ampicillin/sulbactam & 144 & $123(85.4 \%)$ & $21(14.6 \%)$ \\
\hline Penicillin & 144 & $142(98.6 \%)$ & $02(1.3 \%)$ \\
\hline Piperacillin/tazobactam & 144 & $113(78.5 \%)$ & $31(21.5 \%)$ \\
\hline \multicolumn{4}{|l|}{ Cephalosporin group } \\
\hline Cefaclor & 144 & $127(88.2 \%)$ & $17(11.8 \%)$ \\
\hline Cefazolin & 144 & $116(80.6 \%)$ & $28(19.4 \%)$ \\
\hline Cefoperazone or sulbactam & 144 & $136(94.4 \%)$ & $8(5.6 \%)$ \\
\hline Cefoxitin & 144 & $103(71.5 \%)$ & $41(28.5 \%)$ \\
\hline Cefotaxime & 112 & $106(94.6 \%)$ & $6(5.4 \%)$ \\
\hline Ceftriaxone & 143 & $115(80.4 \%)$ & $28(19.6 \%)$ \\
\hline Cefuroxime & 136 & $111(81.6 \%)$ & $25(18.4 \%)$ \\
\hline Cefixime & 143 & $142(99.3 \%)$ & $1(0.7 \%)$ \\
\hline Cefalexin & 144 & $128(88.9 \%)$ & $16(11.1 \%)$ \\
\hline Cefepime & 143 & $137(95.8 \%)$ & $6(4.2 \%)$ \\
\hline \multicolumn{4}{|l|}{ Fluoroquinolone group } \\
\hline Ciprofloxacin & 144 & $88(61.1 \%)$ & $56(38.9 \%)$ \\
\hline Moxifloxacin & 144 & $130(90.3 \%)$ & $14(9.7 \%)$ \\
\hline Lomefloxacin & 144 & $143(99.3 \%)$ & $1(0.7 \%)$ \\
\hline Levofloxacin & 143 & $108(75.5 \%)$ & $35(24.5 \%)$ \\
\hline \multicolumn{4}{|l|}{ Aminoglycoside group } \\
\hline Tobramycin & 144 & $135(93.8 \%)$ & $9(6.2 \%)$ \\
\hline Fosfomycin & 144 & $143(99.3 \%)$ & $1(0.7 \%)$ \\
\hline Gentamicin & 144 & 76 (52.8\%) & $68(47.2 \%)$ \\
\hline Amikacin & 144 & $63(43.8 \%)$ & $81(56.2 \%)$ \\
\hline Monobactam such as aztreonam & 144 & $143(99.3 \%)$ & $1(0.7 \%)$ \\
\hline \multicolumn{4}{|l|}{ Carbapenem group } \\
\hline Invanz ertapenem & 144 & $139(96.5 \%)$ & $5(3.5 \%)$ \\
\hline Imipenem & 144 & $58(40.3 \%)$ & $86(59.7 \%)$ \\
\hline Meropenem & 144 & $111(77.1 \%)$ & $33(22.9 \%)$ \\
\hline Doripenem & 144 & $133(92.4 \%)$ & $11(7.6 \%)$ \\
\hline \multicolumn{4}{|l|}{ Macrolide group } \\
\hline Azithromycin & 144 & $136(94.4 \%)$ & $8(5.6 \%)$ \\
\hline Clarithromycin & 144 & $138(95.8 \%)$ & $6(4.2 \%)$ \\
\hline Clindamycin & 144 & $83(57.6 \%)$ & $61(42.4 \%)$ \\
\hline
\end{tabular}




\section{Cureus}

\begin{tabular}{|c|c|c|c|}
\hline Erythromycin & 144 & $134(93.1 \%)$ & $10(6.9 \%)$ \\
\hline \multicolumn{4}{|l|}{ Other antibiotics } \\
\hline Chloramphenicol & 144 & 61 (42.4\%) & $83(57.6 \%)$ \\
\hline Co-trimoxazole & 144 & 94 (65.3\%) & 50 (34.7\%) \\
\hline Doxycycline & 144 & 68 (47.2\%) & 76 (52.8\%) \\
\hline Vancomycin & 144 & 60 (41.7\%) & 84 (58.3\%) \\
\hline Minocycline & 144 & 76 (52.8\%) & 68 (47.2\%) \\
\hline Rifampicin & 144 & 99 (68.8\%) & 45 (31.2\%) \\
\hline Novobiocin & 144 & $143(99.3 \%)$ & $1(0.7 \%)$ \\
\hline Colistin & 144 & 140 (97.2\%) & $4(2.8 \%)$ \\
\hline Nalidixic acid & 144 & 144 (100\%) & $0.0(0 \%)$ \\
\hline Tigecycline & 144 & 143 (99.3\%) & $1(0.7 \%)$ \\
\hline Nitrofurantoin & 144 & 143 (99.3\%) & $1(0.7 \%)$ \\
\hline Teicoplanin & 144 & 141 (97.9\%) & $3(2.1 \%)$ \\
\hline
\end{tabular}

TABLE 1: Sensitivity and resistance pattern ( $N=$ total number of cultures where antibiotic was tested)

\section{Discussion}

Antibiotic resistance remains a major cause of mortality in bacterial infections across the age spectrum. Septicemia remains a major problem in developing countries including Pakistan. Irrational use of antibiotics has led to high levels of resistance among bacteria responsible for septicemia. Empiric antibacterial therapy should follow from most likely organisms and their known local resistance patterns. The present study provides data on causative organisms and their susceptibilities to commonly used antibacterials. The present study indicates that MRSA was the most common causative organism (37.5\% of sepsis cases). Skin contaminants were found in $17.6 \%$, and MRSA accounted for $14.8 \%$ cases. The result of the present study is consistent with other studies that found Staphylococcus aureus to be the most commonly identified pathogen [15-16]. Studies on adult septic patients indicate that Klebsiella pneumonia (8.14\%) and Escherichia coli (4.65\%) were the most frequently detected bacteria [16]. In contrast, our study showed that Klebsiella was detected in $3.4 \%$ and Escherichia coli in $11.4 \%$.

Resistance and sensitivity pattern reveals that the majority of bacteria were resistant to common antibiotics used in general practices. There was no single antibiotic that has $>70 \%$ susceptibility. Results indicate that in the penicillin group, efficacies of piperacillin/tazobactam and ampicillin/sulbactam were $21.5 \%$ and $14.6 \%$, respectively. This susceptibility proportion was lower than that reported by Pradipta et al. in Indonesia (66.7\% and 60.0\% efficacies for piperacillin/tazobactam and ampicillin/sulbactam, respectively) [17]. In the present study, among the cephalosporins, cefoxitin had the highest susceptibility (28.5\%).

Among fluoroquinolones, only $41.0 \%$ of samples were susceptible to ciprofloxacin and $24.5 \%$ to levofloxacin. The results of the present study were concordant with findings by Pradipta et al [17]. The present study reports global values for sensitivities by antibacterials rather than for each identified organism. Based on published studies, ciprofloxacin resistance is common in Staphylococcus aureus and Enterococci (50\% and $33.3 \%$, respectively). Among gram-negative microorganisms, ciprofloxacin resistance in Acinetobactericia, Salmonella typhi, Escherichia coli, and Pseudomonas aeruginosa against ciprofloxacin is reported as 9.1\%, $6.3 \%, 26.1 \%$, and $20 \%$, respectively [18].

Among aminoglycosides, amikacin and gentamycin were found to be effective in $56.3 \%$ and $47.2 \%$ of septic cases, respectively. There are controversial findings about the resistant pattern of bacteria causing septicemia. A study conducted on bacterial isolate indicates that $55.56 \%$ of gram-negative bacteria were resistant to amikacin [19]. In such condition, it is recommended that aminoglycoside could be given in high dose. A study from Bangladesh among neonates with septicemia indicates that both gentamicin and amikacin have sensitivity pattern against gram-negative and gram-positive bacteria, ranging from $90 \%$ to $100 \%$ [20]. Another study reported that $75.0 \%$ of gram-positive isolates in septic patients show resistance to gentamicin [21]. This indicates that the sensitivity pattern varies across the countries depending on the use of antibiotics, immunity, and status of health care system as a whole. Thus, a local antibiogram broadly 


\section{Conclusions}

Antibacterial resistance among bacteria-causing sepsis was alarmingly widespread. No single agent demonstrated bacterial susceptibility greater than $60 \%$, with the majority being much lower. Possible reasons for this may include excessive use prophylactic antibiotics along and easy availability of over-thecounter drugs without prescriptions. A review on the use and availability of antibiotics is therefore highly recommended.

\section{Additional Information \\ Disclosures}

Human subjects: Consent was obtained or waived by all participants in this study. Animal subjects: All authors have confirmed that this study did not involve animal subjects or tissue. Conflicts of interest: In compliance with the ICMJE uniform disclosure form, all authors declare the following: Payment/services info: All authors have declared that no financial support was received from any organization for the submitted work. Financial relationships: All authors have declared that they have no financial relationships at present or within the previous three years with any organizations that might have an interest in the submitted work. Other relationships: All authors have declared that there are no other relationships or activities that could appear to have influenced the submitted work.

\section{References}

1. Rudd KE, Kissoon N, Limmathurotsakul D, et al.: The global burden of sepsis: barriers and potential solutions. Crit Care. 2018, 22:232. 10.1186/s13054-018-2157-z

2. Sankar J, Dhochak N, Kumar K, Singh M, Sankar MJ, Lodha R: Comparison of International Pediatric Sepsis Consensus Conference versus Sepsis-3 Definitions for children presenting with septic shock to a tertiary care center in India: a retrospective study. Pediatr Crit Care Med. 2019, 20:e122-9. 10.1097/PCC.0000000000001864

3. Rudd KE, Johnson SC, Agesa KM, et al.: Global, regional, and national sepsis incidence and mortality, 19902017: analysis for the Global Burden of Disease Study. Lancet. 2020, 395:200-11. 10.1016/S01406736(19)32989-7

4. Asghar A, Hashmi M, Rashid S, Khan FH: Incidence, outcome and risk factors for sepsis--a two year retrospective study at surgical intensive care unit of a teaching hospital in Pakistan. J Ayub Med Coll Abbottabad. 2016, 28:79-83.

5. Vincent JL, Sakr Y, Sprung CL, et al.: Sepsis in European intensive care units: results of the SOAP study . Crit Care Med. 2006, 34:344-53. 10.1097/01.ccm.0000194725.48928.3a

6. Sohail M, Sultana Q, Rasool K, Sarwar S, Basit A, Khalid M: Bacteremia prediction by inflammatory factors and recent trend in drug resistance of bacteria isolated from blood stream infection. J Inf Mol Biol. 2015, 3:75-80. 10.14737/journal.jimb/2015/3.3.75.80

7. Ullah AR, Hussain A, Ali I, Samad A, Ali Shah ST, Yousef M, Khan TM: A prospective observational study assessing the outcome of sepsis in intensive care unit of a tertiary care hospital, Peshawar. Pak J Med Sci. 2016, 32:688-93. 10.12669/pjms.323.9978

8. Paoli CJ, Reynolds MA, Sinha M, Gitlin M, Crouser E: Epidemiology and costs of sepsis in the United Statesan analysis based on timing of diagnosis and severity level. Crit Care Med. 2018, 46:1889-97. 10.1097/CCM.0000000000003342

9. Starr ME, Saito H: Sepsis in old age: review of human and animal studies . Aging Dis. 2014, 5:126-36 10.14336/AD.2014.0500126

10. Martin GS, Mannino DM, Eaton S, Moss M: The epidemiology of sepsis in the United States from 1979 through 2000. N Engl J Med. 2003, 348:1546-54. 10.1056/NEJMoa022139

11. Randolph AG, McCulloh RJ: Pediatric sepsis: important considerations for diagnosing and managing severe infections in infants, children, and adolescents. Virulence. 2014, 5:179-89. 10.4161/viru.27045

12. Shirazi H, Riaz S, Mahmood RA: Morbidity and mortality pattern of newly born babies in a teaching hospital. J Rawalpindi Med Coll. 2015, 19:204-8.

13. Wattal C, Kler N, Oberoi JK, Fursule A, Kumar A, Thakur A: Neonatal sepsis: mortality and morbidity in neonatal sepsis due to multidrug-resistant (MDR) organisms: part 1. Indian J Pediatr. 2020, 87:117-21. 10.1007/s12098-019-03106-7

14. Sepsis: recognition, diagnosis and early management. NICE guideline [NG51] . (2017). Accessed: April 1, 2019: https://www.nice.org.uk/guidance/ng51/chapter/Recommendations\#identifying-people-withsuspected-sepsis.

15. Negussie A, Mulugeta G, Bedru A, Ali I, Shimeles D, Lema T, Aseffa A: Bacteriological profile and antimicrobial susceptibility pattern of blood culture isolates among septicemia suspected children in selected hospitals Addis Ababa, Ethiopia. Int J Biol Med Res. 2015, 6:4709-17.

16. Goswami NN, Trivedi HR, Goswami AP, Patel TK, Tripathi CB: Antibiotic sensitivity profile of bacterial pathogens in postoperative wound infections at a tertiary care hospital in Gujarat, India. J Pharmacol Pharmacother. 2011, 2:158-64.

17. Pradipta IS, Sodik DC, Lestari K, Parwati I, Halimah E, Diantini A, Abdulah R: Antibiotic resistance in sepsis patients: evaluation and recommendation of antibiotic use. N Am J Med Sci. 2013, 5:344-52. 10.4103/19472714.114165

18. Alam MS, Pillai PK, Kapur P, Pillai KK: Resistant patterns of bacteria isolated from bloodstream infections at a university hospital in Delhi. J Pharm Bioallied Sci. 2011, 3:525-30. 10.4103/0975-7406.90106 


\section{Cureus}

19. Meyer RD, Lewis RP, Finegold SM: Amikacin therapy of serious gram-negative bacillary infections in chronic hemodialysis patients. Chemotherapy. 1978, 24:172-8. 10.1159/000237778

20. Afrin M, Siddique MA, Ahmed AA, et al.: Neonatal septicemia: isolation, identification and antibiotic sensitivity pattern of bacteria in a tertiary hospital in Bangladesh. Faridpur Med Coll J. 2016, 11:58-61.

21. Kitila KT, Taddese BD, Hailu TK, et al.: Assessment of bacterial profile and antimicrobial resistance pattern of bacterial isolates from blood culture in Addis Ababa Regional Laboratory, Addis Ababa, Ethiopia. Clin Microbiol. 2018, 7:1-6 